\title{
Effects of dietary fiber on postprandial glucose in healthy adults
}

\author{
F Yesmin ${ }^{1 凶}$, MOI Ali ${ }^{2}$, MMR Sardar ${ }^{3}$, MK Munna ${ }^{4}$, S Baksh $^{5}$
}

\begin{abstract}
Frequently and persistently raised glucose level causes numerous complications. So it is important to find out a component of diet that can improve glucose homeostasis after ingestion of food without causing any side effect or complication. The aim of this randomized controlled clinical trial study was to verify the effect of dietary fiber on postprandial blood sugar (PPBS). The study was carried out in a sample of fifty healthy adult subjects at the Department Physiology of Rajshahi Medical College during the period from January 2014 to December 2014. Fasting blood sugar of the subjects was estimated before ingestion of identical glucose drink with or without dietary fiber (ispaghula husk) and PPBS was measured at 30, 60 and 120 min after the drink. The mean plasma glucose concentrations after a $50 \mathrm{gm}$ oral glucose load significantly $(p<0.05, p<0.001)$ decreased at 30,60 and 120 min in both condition with dietary fiber and without dietary fiber. The mean plasma postprandial glucose concentration in condition with dietary fiber was significantly $(p<0.001)$ lower than the corresponding value without dietary fiber. The study suggests that ingestion of water soluble dietary fiber along with glucose drink reduces sharp rise as well as sustained rise of postprandial glucose. Key words: dietary fiber, ispaghula husk, postprandial glucose, healthy subjects.
\end{abstract}

\section{Introduction}

Glucose is the only nutrient that normally can be used by the brain, retina and germinal epithelium of the gonads in sufficient quantities to supply them optimally with their required energy. Therefore, it is important to maintain the blood glucose concentration at a sufficiently high level to provide this necessary nutrition. However, long term increase in blood glucose may cause damage to many tissues, especially to blood vessels. Vascular injury associated with uncontrolled diabetes mellitus leads to increased risk for heart attack, stroke, end stage renal disease and blindness. ${ }^{1}$
Dietary fibers are highly complex substances that can be described as any non-digestible food plant carbohydrates and lignins in which the plant matrix is largely intact. Soluble dietary fibers are plant foods such as legumes, oat, barley, some fruits, vegetables, psyllium seed husk, nuts, almonds, etc. ${ }^{2}$ Hlebowicz et al failed to find out any effect of dietary fiber on postprandial blood sugar (PPBS). ${ }^{3}$ On the contrary, Johansson et al and Nilsson et al stated that ingestion of resistant starch reduced the PPBS during the course of the entire experimental day. ${ }^{4,5}$ Karhunen et al noted that the addition of soluble dietary fiber to the test

\footnotetext{
1. F Yesmin, MBBS, MPhil, Assistant Professor of Physiology, Diabetic Association Medical College, Faridpur. Email: y.farjana22@gmail.com

2. MOI Ali, MBBS, MPhil, Associate Professor of Physiology, Rajshahi Medical College, Rajshahi

3. MMR Sardar, MBBS, MPhil, Assistant Professor of Physiology, Khulna Medical College, Khulna

4. MK Munna, MBBS, MPhil, Lecturer of Physiology, Rajshahi Medical College, Rajshahi

5. S Baksh, MBBS, MPhil, Lecturer of Physiology, Cox's Bazar Medical College, Cox's Bazar
} 
meals strongly decreased the PPBS response compared with the low fiber meals. ${ }^{6}$ Following ingestion of glucose drink, Sierra et al found significant effect of dietary fiber on PPBS but Jarjis et al failed to find out any effect. 7,8 So, the studies have produced conflicting results regarding the effect of dietary fiber on PPBS.

As the concentration of blood glucose rises above $100 \mathrm{mg} / \mathrm{dl}$ of blood, the rate of insulin secretion rises rapidly, reaching a peak some 10 to 25 times the basal level at blood glucose concentrations between 400 and $600 \mathrm{mg} / \mathrm{dl}$. Thus, the increase in insulin secretion under a glucose stimulus is dramatic both in its rapidity and in the tremendous level of secretion achieved. Furthermore, the turn-off insulin secretion is almost equally as rapid, occurring within 3 to 5 minutes after reduction in blood glucose concentration back to the fasting level. ${ }^{1}$

This response of insulin secretion to an elevated blood glucose concentration provides an extremely important feedback mechanism for regulating blood glucose concentration. That is, any rise in blood glucose increases insulin secretion and the insulin in turn increases transport of glucose into liver, muscle, and other cells, thereby reducing the blood glucose concentration back toward the normal value. ${ }^{1}$

In a normal person the blood glucose concentration is narrowly controlled, usually between 80 to $90 \mathrm{mg} / \mathrm{dl}$ in the fasting person each morning before breakfast. This concentration increases to 120 to $140 \mathrm{mg} / \mathrm{dl}$ during the first hour or so after a meal, but the feedback system for control of blood glucose return the glucose concentration rapidly back to the control level, usually within 2 hours after the last absorption of carbohydrates. In persons with abnormal glucose metabolism, after ingestion of meal, it takes about 4 to 6 hours to reach the normal blood glucose level following the rise. ${ }^{1}$

So, ingestion of food causes sharp rise of glucose as well as sustained rise of glucose in normal persons and in persons with abnor- mal glucose metabolism. As a result, it is necessary to find out a component of diet which maintains glucose homeostasis without any side effect or complication. Therefore, the aim of the study was to evaluate the effect of dietary fiber on postprandial glucose concentration in healthy adults.

\section{Materials and Method}

This present study was carried out in the Department of Physiology, Rajshahi Medical College between the periods of January 2014 to December, 2014. Fifty healthy adults aged 18-35 years of both sexes were enrolled in study group. A systematic random sampling technique was applied to select each study subject from medical students and doctors of Rajshahi Medical College and the protocol of this study was approved by Ethical Review Committee of Rajshahi Medical College.

All the subjects were free from diabetes, pregnancy, alcoholism, liver, renal and endocrine disorders. Before enrollment, aim, benefit and procedure of the study was explained and informed written consent was taken from the participants. Thorough physical examination of all subjects was done. On the first assay and after an overnight fast, the subjects took $50 \mathrm{gm}$ glucose dissolved in $250 \mathrm{ml}$ water. On the second assay, the subjects ingested identical glucose drink, supplemented with $10.5 \mathrm{gm}$ ispaghula by stirring as soon as possible. We gave identical glucose load to all subjects, the most easily absorbable form of carbohydrate. Moreover, we used $10.5 \mathrm{gm}$ of ispaghula because it is the maximum daily recommended dose.

Under aseptic precaution, venous blood samples were taken from antecubital vein at fasting and after 30, 60 and 120 min after glucose intake. Plasma was separated by ultracentrifugation. Fasting and postprandial glucose levels were measured by glucose oxidase method using a colorimeter.

Data were analyzed by a computer using SPSS software program. Statistical analysis was done by Student's $t$-test or paired $t$-test 
Table 1. Baseline characteristics of the study subjects, $n=50$

\begin{tabular}{lrr}
\hline Variable & Mean \pm SD & Range \\
\hline Age $(\mathrm{yrs})$ & $23.9 \pm 4.1$ & $20-35$ \\
Weight $(\mathrm{kg})$ & $61.1 \pm 7.7$ & $42-72$ \\
Height $(\mathrm{cm})$ & $1.6 \pm 0.1$ & $1.5-1.8$ \\
BMI $\left(\mathrm{kg} / \mathrm{m}^{2}\right)$ & $22.3 \pm 1.5$ & $18.7-24.6$ \\
Pulse (beats/minute) & $71.0 \pm 4.2$ & $62-80$ \\
Systolic blood pressure $(\mathrm{mmHg})$ & $114.8 \pm 7.8$ & $100-125$ \\
Diastolic blood pressure $(\mathrm{mmHg})$ & $74.2 \pm 7.0$ & $60-80$ \\
\hline
\end{tabular}

n, number.

Table 2. Plasma glucose concentrations ( $\mathrm{mmol} / \mathrm{l})$ after a $50 \mathrm{gm}$ oral glucose load, $\mathbf{n}=\mathbf{5 0}$

\begin{tabular}{lcc}
\hline Variable & $\begin{array}{c}\text { Without dietary fiber } \\
\text { Mean } \pm \text { SD }\end{array}$ & $\begin{array}{c}\text { With dietary fiber } \\
\text { Mean } \pm \text { SD }\end{array}$ \\
\hline Fasting blood sugar & $4.33 \pm 0.50$ & $4.40 \pm 0.56$ \\
Postprandial blood sugar after $30 \mathrm{~min}$ & $8.28 \pm 0.74^{* *}$ & $6.46 \pm 0.63^{* *}$ \\
Postprandial blood sugar after $60 \mathrm{~min}$ & $6.75 \pm 0.61^{* *}$ & $5.68 \pm 0.66^{* *}$ \\
Postprandial blood sugar after $120 \mathrm{~min}$ & $5.24 \pm 0.59^{* *}$ & $4.60 \pm 0.55^{*}$
\end{tabular}

n, number. ${ }^{* *}: p<0.001,{ }^{*}: p<0.05$ by Student's paired $t$-test.

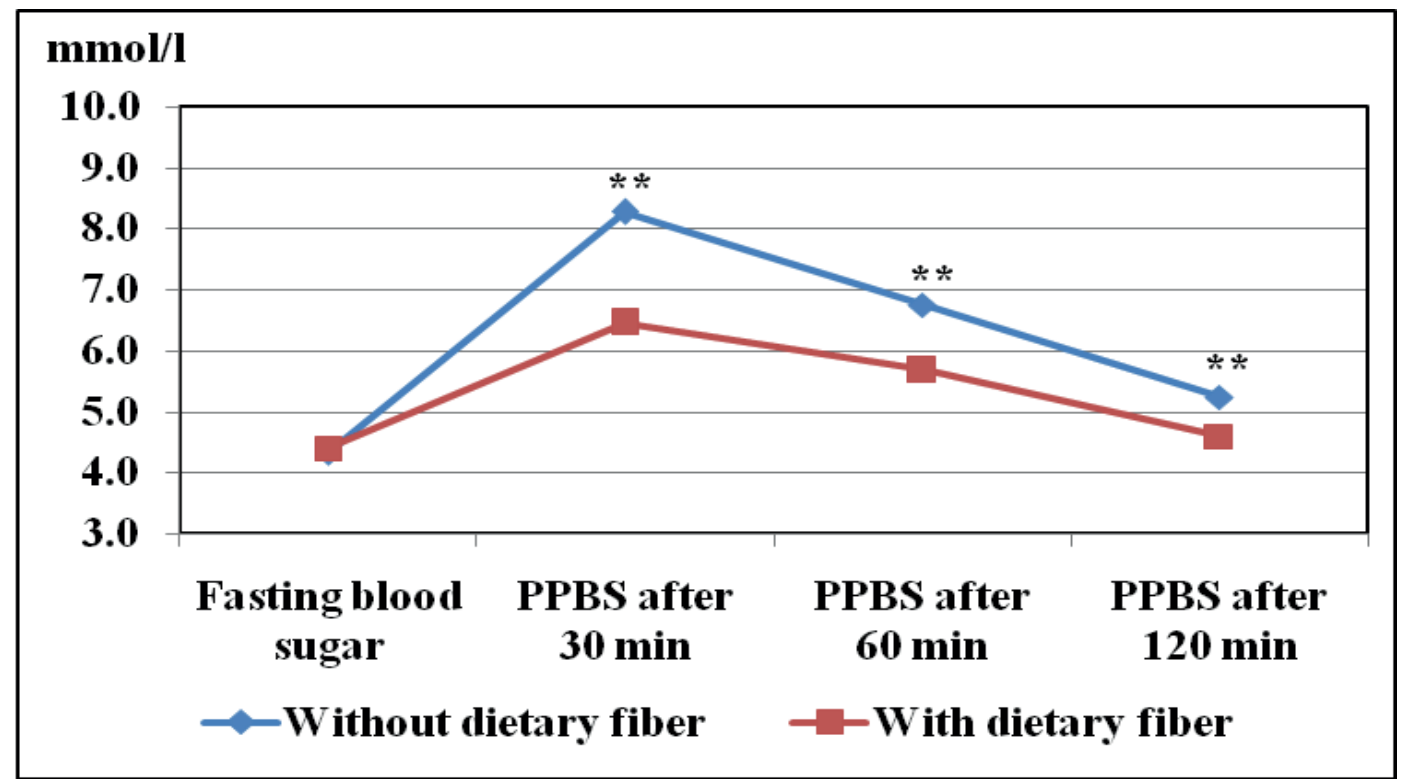

Fig. 1. Plasma glucose concentrations $(\mathrm{mmol} / \mathrm{l})$ after a $50 \mathrm{gm}$ oral glucose load, $\mathbf{n}=5$ 0. PPBS, postprandial blood sugar, ${ }^{* *}: p<0.001$ by Student's $t$-test.

and a $p$ value $<0.05$ was taken as significant.

\section{Results}

Table 1 shows the baseline characteristics of the study subjects. The mean BMI of the subjects was within normal range. All of them also had their blood pressure within normal range. Table 2 shows the plasma glucose concentrations $(\mathrm{mmol} / \mathrm{l})$ after a $50 \mathrm{gm}$ oral glucose load. The mean plasma postprandial glucose concentration significantly $(p<0.05$, $p<0.001$ ) decreased at 30,60 and $120 \mathrm{~min}$ in both condition with dietary fiber and without dietary fiber. However, the mean plasma postprandial glucose concentration at 30,60 and $120 \mathrm{~min}$ in condition with dietary fiber 
was significantly $(p<0.001)$ lower than the corresponding value without dietary fiber (Fig. 1).

\section{Discussion}

Composition and physiochemical properties of food in a meal are important in regulating the glycemic response. Low dietary fiber intake is associated with high incidence of diabetes, colon cancer, coronary heart disease, obesity, hypertension etc. The aim of the present study was to evaluate the effect of soluble dietary fiber on postprandial glucose concentration. Fasting blood sugar of the subjects was estimated before ingestion of identical glucose drink with or without dietary fiber (ispaghula husk) and PPBS was measured at 30,60 and 120 min after the drink. The mean plasma glucose concentrations after a $50 \mathrm{gm}$ oral glucose load significantly decreased at 30,60 and $120 \mathrm{~min}$ in both condition with dietary fiber and without dietary fiber.

In this study, the mean plasma postprandial glucose concentration in condition with dietary fiber was significantly lower than the corresponding value without dietary fiber. This observation is compatible with Sierra et al. ${ }^{7}$ It may be due to the local effect of dietary fiber on glucose absorption, because dietary fiber itself is neither digested nor absorbed. The water soluble dietary fiber dissolves in water and forms viscous gels. The viscosity delays the gastric emptying and slows the rate of intestinal absorption of glucose by forming an effective unstirred layer in the intestinal wall. ${ }^{7}$ However, Jarjis et al failed to find out any significant effect of dietary fiber on PPBS at 30 minute and 60 minute. ${ }^{8}$ Their results were different from our because we have given higher dose of dietary fiber than them. Moreover, they stirred the ispaghula husk into the glucose solution and then allowed the mixture to stand at room temperature overnight to become fully hydrated. Perhaps the procedure altered the rheological property of the mixture.

In this study, we found that the dietary fiber significantly reduced the PPBS even two hours after glucose drink. It indicates that dietary fiber especially ispaghula husk have long lasting effect on blood glucose level. This observation is compatible with Nilsson et al observed that specific indigestible carbohydrates can modify the glucose response not only in the acute phase but also during the course of a whole day. ${ }^{5}$

We also found that the mean plasma postprandial glucose concentration in condition with dietary fiber was significantly lower than the corresponding value without dietary fiber. Ulmius et al stated that meals with rye bran containing soluble fiber gave a lower postprandial glucose peak when compared with the control meal. ${ }^{9}$ This observation is in agreement with the finding of the present study, which indicated that consumption of soluble dietary fiber resulted in decreased postprandial glucose. However, Chiu and Steward found no significant difference between the glycaemic indices of high and low resistant starch consumption. ${ }^{10}$ It is possible that the difference in resistant starch content between high and low resistant starch diet was not sufficient enough to cause difference in postprandial glycaemic response. It is our assumption that ingestion of dietary fiber along with meal will reduce the requirement of preformed insulin as well as newly synthesized insulin during postprandial period.

One of the strength of the present study is that it is a true experimental study with dietary fiber intervention. Medical intervention was done at short interval in same individuals in same experimental setting to avoid bias. However, we did not measure fiber viscosity and blood insulin concentration which may be the limitation of the study.

It was a cross sectional study on small sample size. So, a study on larger sample size should be done to confirm the findings. Moreover, effect of dietary fiber on digestion of food should be assessed by administering dietary fiber along with test meal instead of glucose drink.

\section{Conclusion}

The study proved that dietary fiber ingestion 
prevented sharp rise as well as sustained rise of blood glucose. Therefore, dietary fiber seems to be a suitable component for the treatment of patients with abnormal glucose metabolism. Moreover, it should be an essential component of diet of healthy adults.

\section{Conflict of interest: None.}

\section{References}

1. Hall J, Hall J. Guyton and Hall Textbook of Medical Physiology. 12th ed. Philadelphia: Saunders-Elsevier, 2011.

2. James $M$, Lattimer D, Haub. Effects of dietary fiber and its components on metabolic health. Nutrients 2010;2(12):1266-89.

3. Hlebowicz J, Jönsson JM, Lindstedt S, Björgell O, Darwich G, Almér LO. Effect of commercial rye whole-meal bread on postprandial blood glucose and gastric emptying in healthy subjects. Nutr $\mathrm{J}$ 2009;8:1-5.

4. Johansson EV, Nilsson AC, Östman EM, Björck IM. Effect of indigestible carbohydrates in barley on glucose metabolism, appetite and voluntary food intake over $16 \mathrm{~h}$ in healthy adults. Nutr J 2013;12:46.

5. Nilsson AC, Östman EM, Granfeldt $Y$, Björck IM. Effect of cereal test breakfasts differing in glycemic index and content of indigestible carbohydrates on daylong glucose tolerance in healthy subjects. Am J Clin Nutr 2008;87:645-54.

6. Karhunen LJ, Juvonen KR, Flander SM, et al. A psyllium fiber-enriched meal strongly attenuates postprandial gastrointestinal peptide release in healthy young adults. J Nutr 2010;140(4):73744.

7. Sierra M, Garcia JJ, Fernández N, et al. Effects of ispaghula husk and guar gum on postprandial glucose and insulin concentrations in healthy subjects. Eur $\mathrm{J}$ Clin Nutr 2001;55(4):235-43.

8. Jarjis HA, Blackburn NA, Redfern JS, Read NW. The effect of ispaghula (Fybogel and Metamucil) and guar gum on glucose tolerance in man. $\mathrm{Br} \mathrm{J}$ Nutr 1984;51(3):371-8.

9. Ulmius $M$, Johansson A, Onning G. The influence of dietary fibre source and gender on the postprandial glucose and lipid response in healthy subjects. Eur $\mathrm{J}$ Nutr 2009;48(7):395-402.

10.ChiuYT, Stewart ML. Effect of variety and cooking method on resistant starch content of white rice and subsequent postprandial glucose response and appetite in humans. Asia Pac J Clin Nutr 2013;22(3):372-9.

\section{Suggestion for citation of the above:}

Yesmin F, Ali MOI, Sardar MMR, Munna MK, Baksh S. Effects of dietary fiber on postprandial glucose in healthy adults. Mediscope 2019;6(1):25-9. 